PROCEEDINGS OF THE

AMERICAN MATHEMATICAL SOCIETY

Volume 129, Number 3, Pages 629-637

S 0002-9939(00)05738-5

Article electronically published on September 20, 2000

\title{
SEMIDIRECT SUM OF GROUPS IN WHICH ENDOMORPHISMS ARE GENERATED BY INNER AUTOMORPHISMS
}

\author{
FENG-KUO HUANG
}

(Communicated by Stephen D. Smith)

\begin{abstract}
An I-E group is a group $G$ in which every endomorphism is finitely generated by its inner automorphisms. In this paper a characterization for a semidirect sum of I-E groups to be an I-E group is obtained and some wellknown results are generalized. We then use this characterization to prove that a semidirect sum of finite I-E groups will again be an I-E group if the normal semidirect summand is unique and fully invariant. Conditions for a group to be an I-E group are also given.
\end{abstract}

\section{INTRODUCTION}

A group $G$ is called an I-E group if all endomorphisms of $G$ are generated by inner automorphisms. $\operatorname{Inn}(G)$ and $\operatorname{End}(G)$ denote the set of all inner automorphisms and endomorphisms of $G$, respectively. The group operation is denoted additively even when $G$ is not necessarily abelian. Consequently, we use semidirect sum instead of semidirect product. Herein we use the right-hand mapping convention: $a(f g)=(a f) g$ for all $a \in G$. The terminology used in this paper follows Meldrum [18] and Robinson [21.

The study of I-E groups can be traced back to at least two different origins. In 1963, L. Fuchs [9] had raised the following question: For which abelian groups $G$ do their automorphism groups Aut $(G)$ generate the endomorphism ring $\operatorname{End}(G)$ ? R. S. Pierce [20, R. W. Stringall [23], H. Freedman [7] and F. Castagna [3] gave certain results on both the positive and the negative sides of this question. During the same period, A. Fröhlich [8] had shown that finite simple groups are I-E groups. The next step in this direction was taken by J. J. Malone and C. G. Lyons; they showed that a dihedral group $D_{n}$ of order $2 n$ is an I-E group only if $n$ is odd [12, 13]. In a further step, J. J. Malone had shown that the generalized quaternion groups $Q_{n}$ are not I-E groups [15. These investigations were recently generalized by C. G. Lyons and G. Mason in [14]; they proved that dicyclic groups of order $4 n$ with $n$ odd are I-E groups. Next Y. Fong and J. D. P. Meldrum proved that symmetric groups $S_{n}$ with $n>4$ are I-E groups [5, 6]. In 22], G. Saad, M. J. Thomsen, and S. A. Syskin claimed alternating groups $A_{n}$ with $n \neq 4$, and special linear groups $S L(n, q)$, except $S L(2,3)$, are all I-E groups. For a detailed history of I-E groups, refer either to [16] or [22].

Received by the editors May 7, 1999.

2000 Mathematics Subject Classification. Primary 16Y30; Secondary 20 E36.

Key words and phrases. Endomorphism near-ring, I-E group.

(C)2000 American Mathematical Society 
The above results primarily determine some concrete examples for which a group $G$ is I-E. Recently, questions concerning the structure of I-E groups have been considered. J. J. Malone and G. Mason [17] have shown: a semidirect sum of cyclic groups of relatively prime order is I-E when the cyclic normal semidirect summand is the commutator subgroup. C. G. Lyons and G. L. Peterson [10] then made the following improvement: a semidirect sum of cyclic groups of relatively prime order is $I-E$. Observing that a finite abelian group is $\mathrm{I}-\mathrm{E}$ if and only if it is cyclic, S. A. Syskin [25] proves: a semidirect sum of two I-E groups of relatively prime orders is an $I-E$ group.

Their results depend heavily on [10 Theorem 2.1], which characterizes when a semidirect product of I-E groups of relatively prime orders is an I-E group. In this paper, not assuming $G$ to be finite, this characterization theorem is generalized and, at the same time, a fairly concise proof is provided in Theorem 2.1. We then use this result, in Theorem 2.11, to prove that a semidirect sum of I-E groups will be an I-E group if the normal semidirect summand is fully invariant and is a unique minimal normal subgroup. Some conditions to ensure that a group will be an I-E group are also given in Proposition 2.10 and in Theorem 3.4. Moreover, we briefly discuss the direct sum of I-E groups. Using Theorem 3.4, we generalize another result by C. G. Lyons and G. L. Peterson in Corollary 3.5. Examples are provided to illustrate and delimit our results.

The near-ring generated by the group of inner automorphisms $\operatorname{Inn}(G)$ is denoted by $I(G)$, and $E(G)$ will denote the near-ring generated by the endomorphisms $\operatorname{End}(G)$. For a group $G$ and its subgroups $H$ and $K$, the centralizer of $H$ in $K$ will be denoted as $C_{K}(H)$. Let $I(G, H)=\{f \in I(G) \mid G f \subseteq H\}$ and $E(G, H)=$ $\{f \in E(G) \mid G f \subseteq H\}$. Moreover $\mathcal{J}_{2}(N)$ denotes the $\mathcal{J}_{2}$ radical of the near-ring $N$. Details about the $\mathcal{J}_{2}$ radical can be found in [18].

\section{Semidirect sums of I-E Groups}

Recall the $I(G)$-subgroups [18, p. 157] are equivalent to normal subgroups of $G$, and the $E(G)$-subgroups are equivalent to the fully invariant subgroups of $G$. Therefore a necessary condition for $G$ to be an I-E group is that each normal subgroup must be fully invariant. It can easily be shown that this condition is equivalent for a group to be an I-E group when $G$ is finitely generated abelian. Unfortunately, this condition is not sufficient in general. For example, the group $A_{4}$ satisfies this condition but fails to be an I-E group [22]. The following result characterizes the I-E property for a group, which is a semidirect sum of a fully invariant subgroup $H$ and a subgroup $K$, in terms of the behavior of the projection map.

Theorem 2.1. Let $G$ be semidirect sum of a fully invariant subgroup $H$ and $a$ subgroup $K$ of $G$. Suppose $H$ and $K$ are both $I-E$ groups. Then the following are equivalent:

(1) $G$ is an $I-E$ group.

(2) $I(G, H)=E(G, H)$.

(3) The projection map $p: G \rightarrow K$ is in $I(G)$ and $p \alpha(1-p) \in I(G)$ for all $\alpha \in \operatorname{End}(G)$.

Proof. (1) implies (2) and (2) implies (3) are clear. We want to show that (3) implies (1). 
Let $\alpha \in \operatorname{End}(G)$ and write $\alpha=(1-p) \alpha+p \alpha$ where 1 denotes the identity map of $G$. Since $H$ is fully invariant, $H \alpha \subseteq H$. Thus

$$
\left.\alpha\right|_{H}=\left.(1-p) \alpha\right|_{H} \in \operatorname{End}(H) .
$$

Since $H$ is an I-E group, we have $\left.(1-p) \alpha\right|_{H}=\tilde{r}$ for some $\tilde{r} \in I(H)$. Because $\tilde{r}=\sum_{i=1}^{n} \varepsilon_{i} \rho_{h_{i}}$ where each $\rho_{h_{i}}$ is an inner-automorphism induced by $h_{i} \in H$ and $\varepsilon_{i} \in\{1,-1\}$ for $i \in\{1,2, \ldots, n\}$, we may view $\tilde{r}=\left.r\right|_{H}$ where $r=\sum_{i=1}^{n} \varepsilon_{i} \rho_{h_{i}} \in I(G)$. Note that $1-p \in I(G)$ by our hypothesis and hence $(1-p) r \in I(G)$. It follows that $(1-p) \alpha=(1-p) r \in I(G)$.

Now, we need to show $p \alpha \in I(G)$. Write $p \alpha=p \alpha(1-p)+p \alpha p$. It suffices to show that $p \alpha p \in I(G)$. Note that $p \alpha p$ is an endomorphism of $G$ and $G p \alpha p \subseteq K$. Therefore $\left.p \alpha p\right|_{K} \in \operatorname{End}(K)$.

By using an argument similar to that used above to prove $(1-p) \alpha \in I(G)$, we obtain $p \alpha p \in I(G)$. Therefore $p \alpha \in I(G)$ and $E(G)=I(G)$ as desired.

The following example shows that in Theorem 2.1 the requirement $p \alpha(1-p) \in$ $I(G)$ is indeed necessary.

Example 2.2. Let $G=A_{5} \oplus \mathbb{Z}_{5}$. Observe that both $A_{5}$ and $\mathbb{Z}_{5}$ are I-E groups and that $A_{5}$ is a fully invariant subgroup of $G$. In fact, $A_{5}$ is the commutator subgroup of $G$. From [25, Theorem 3], we know the projection map $p: G \rightarrow \mathbb{Z}_{5}$ is in $I(G)$. However, the order of $E(G)$ is $59^{59} \cdot 5 \cdot 60^{240}$ and the order of $I(G)$ is $59^{59} \cdot 5$ by using the results presented in [24]. Therefore $G$ is not an I-E group.

Corollary 2.3. Let $G$ be semidirect sum of a fully invariant subgroup $H$ and $a$ subgroup $K$ of $G$. Let $p: G \rightarrow K$ be the projection map. Suppose $H$ and $K$ are both $I-E$ groups and $\{p\} \cup \mathcal{J}_{2}(E(G)) \subseteq I(G)$. Then $G$ is an $I-E$ group.

Proof. It is routine to verify that $p \alpha(1-p) \beta$ is nilpotent for all $\alpha, \beta \in E(G)$. Since $\mathcal{J}_{2}(E(G))$ contains all nil right $E(G)$-subgroups [18, Corollary 5.24], $\mathcal{J}_{2}(E(G))$ must contain $p \alpha(1-p) E(G)$, and in particular contains all the elements $p \alpha(1-p)$ for all $\alpha \in \operatorname{End}(G)$. By Theorem 2.1, $G$ is an I-E group.

A 0 -symmetric near-ring $N$ is called 2-primal if the prime radical $\mathcal{P}_{0}(N)$ is equal to the completely prime radical $\mathcal{P}_{2}(N)$. Examples for 2-primal near-rings are abundant. Let $G$ be finite dihedral group with order not divisible by 4 or the generalized quaternion group. Then $E(G)$ is 2-primal. For more details, please refer to [1, 2].

Corollary 2.4. Let $G$ be semidirect sum of a fully invariant subgroup $H$ and a subgroup $K$ of $G$. Let $p: G \rightarrow K$ be the projection map. Suppose $H$ and $K$ are both $I-E$ groups and $E(G)$ is 2-primal. If $\{p\} \cup \mathcal{P}_{0}(E(G)) \subseteq I(G)$, then $G$ is an $I-E$ group.

Proof. Since $\mathcal{P}_{0}(E(G))$ contains all the nilpotent elements including $p \alpha(1-p)$ for all $\alpha \in \operatorname{End}(G)$, the assertion follows from Theorem 2.1.

The utility of Corollary 2.3 and Corollary 2.4 can be readily demonstrated by considering $E\left(S_{3}\right)$ where $S_{3}$ is the symmetric group of order 6 . Observe that $\{p\} \cup$ $\mathcal{J}_{2}\left(E\left(S_{3}\right)\right) \subseteq I\left(S_{3}\right)$ and $E\left(S_{3}\right)$ is 2-primal with $\{p\} \cup \mathcal{P}_{0}\left(E\left(S_{3}\right)\right) \subseteq I\left(S_{3}\right)$ [11]. Hence $E\left(S_{3}\right)$ illustrates both Corollary 2.3 and 2.4. As a corollary, we obtain one of the main results of C. G. Lyons and G. L. Peterson [10]. 
Corollary 2.5 ([10, Theorem 2.1]). Suppose that $G$ is the semidirect sum of a normal subgroup $H$ and a subgroup $K$ where $(|H|,|K|)=1$ and $H, K$ are both $I-E$ groups. Then the following are equivalent:

(1) $G$ is an $I-E$ group.

(2) The projection map $p: G \rightarrow K \in I(G)$.

Proof. Let $\pi$ be the set of prime factors of $|H|$. The hypothesis that $(|H|,|K|)=1$ implies that $G$ is a Hall $\pi$-separable group and $H$ is the unique Hall $\pi$-subgroup of $G$. From Theorem 9.1.6 in 21] and the fact that the homomorphic image of a $\pi$-subgroup is a $\pi$-subgroup, we see that $H$ is a fully invariant subgroup of $G$. Observe that $p \alpha(1-p)=0$ for all $\alpha \in \operatorname{End}(G)$ when the order of $H$ and $K$ are relatively prime. By applying Theorem 2.1 above, we obtain the result.

The following example shows that Theorem 2.1 is a proper generalization of the Lyons-Peterson result [10, Theorem 2.1].

Example 2.6. Let $G=A_{8} \oplus P S L(3,4)$ where $A_{8}$ is the alternating group of degree 8 and $P S L(3,4)$ is the projective special linear group of order $20160=8 ! / 2$. Note that $\operatorname{PSL}(3,4)$ is not isomorphic to $A_{8}$; for it can be demonstrated that $\operatorname{PSL}(3,4)$ has no elements of order 15 , unlike $A_{8}$ which has $(1,2,3,4,5)(6,7,8)$, an element of order 15. Let $p: G \rightarrow P S L(3,4)$ be the projection map. Observe that both $A_{8}$ and $\operatorname{PSL}(3,4)$ are fully invariant subgroups of $G$. Hence $p \alpha(1-p)=0 \in I(G)$ for all $\alpha \in \operatorname{End}(G)$. The restriction map $\left.p\right|_{P S L(3,4)}$ is an endomorphism of $\operatorname{PSL}(3,4)$ which is an I-E group. Therefore $\left.p\right|_{P S L(3,4)} \in I(P S L(3,4))$. On the other hand, $\left.p\right|_{A_{8}}=0 \in I\left(A_{8}\right)$ and so $p \in I(G)$. Hence $G$ is an I-E group by Theorem 2.1. Note that the orders of $A_{8}$ and $P S L(3,4)$ are not relatively prime. In fact, they have the same order.

Let $G$ be a semidirect sum of a normal subgroup $H$ and a subgroup $K$. From 19, Lemma 4.1], we know that $K$ being an I-E group is necessary for $G$ to be an I-E group. In Example 2.7, we exhibit a group $G$ such that $H$ is fully invariant in $G$ and $K$ is an I-E group with $(|H|,|K|)=1$. Moreover, the projection map $p: G \rightarrow K$ is in $I(G)$, but $G$ is not an I-E group. Therefore assuming that $H$ is an I-E group in Corollary 2.5 is not superfluous. However, Example 2.8 shows that, in general, $G$ can be an I-E group with $H$ fully invariant, but $H$ is not an I-E group.

Example 2.7. We first quote a result from [22, Theorem 16]: Let $G$ have a minimal normal subgroup $H$ of order $p^{n}$ for some prime $p$ and $n \geq 1$ such that $C_{G}(H)=H$ and $G / H$ is cyclic of order $q$ prime to $p$. Then $I(G)=E(G)$ if and only if $n=1$.

Let $H \cong \bigoplus_{i=1}^{n} \mathbb{Z}_{2}$ where $n \neq 1$ and $K=\mathbb{Z}_{3}$. Let $G$ be the semidirect sum of $H$ with $K$. $G$ is not an I-E group. Here it can be seen that $H$ is a fully invariant subgroup of $G$ and $K$ is an I-E group. But $H \cong \bigoplus_{i=1}^{n} \mathbb{Z}_{2}$ is not an I-E group when $n \neq 1$. Note that the projection map $p: G \rightarrow K$ is in $I(G)$ by [25, Theorem 2].

Example 2.8. Let $G$ be the symmetric group $S_{4}$ of degree 4 and consider $S_{4}$ as the semidirect sum of the alternating group $A_{4}$ and the cyclic group $\mathbb{Z}_{2} . S_{4}$ is an I-E group but the fully invariant subgroup $A_{4}$ is not an I-E group.

Using the condition of relatively prime on the order of a subgroup and its index, Proposition 2.10 shows that the I-E property can be lifted from a maximal normal subgroup. Note that Proposition 2.10 can be deduced from [25, Theorem 2]. We will provide a detailed constructive proof for easier reference and hopefully motivate 
some clue to improve this result. The following lemma was quoted from [25] which we will use in Proposition 2.10.

Lemma 2.9 ([25, Theorem 1]). Let $G$ be a finite group with a unique minimal normal subgroup $H$. Assume that $G$ is a semidirect sum of $H$ and a subgroup $K$. Then the projection map $p: G \rightarrow K$ is in $I(G)$.

Proposition 2.10. Let $G$ be a finite group with a maximal normal subgroup $H$ such that the order of $H$ is coprime to its index. If $H$ is an $I-E$ group, then $G$ is an $I-E$ group.

Proof. By using the Schur-Zassenhaus theorem, we know the complement $K$ of $H$ in $G$ exists. Since $G / H$ is simple, it is an I-E group. From Corollary 2.5, we can conclude that $G$ is an I-E group if the projection map $p: G \rightarrow K$ is in $I(G)$.

Note that the centralizer of $H$ in $K$, denoted $C_{K}(H)$, is 0 or $K$ by the maximality of $H$. If $C_{K}(H)=K$, then $G=H \oplus K$ with $(|H|,|K|)=1$. Since both $H$ and $K$ are I-E groups, $G$ is an I-E group by [10, Corollary 2.2].

So assume $C_{K}(H)=0$. If $H$ is also a minimal normal subgroup, then it is unique and so $p \in I(G)$ by Lemma 2.9. Suppose $H$ is not minimal. Let $0=H_{n} \subseteq H_{n-1} \subseteq$ $\cdots \subseteq H_{0}=H$ be a principal series of $H$. Since $H$ is an I-E group, each $H_{i}$ is a fully invariant subgroup of $H$ and thus a normal subgroup of $G$ for all $i=0,1, \cdots, n$. Therefore $H_{n-1}$ is a minimal normal subgroup of $G$.

We now want to show that $p_{n-1}: H_{n-1}+K \rightarrow K$ is in $I\left(H_{n-1}+K\right)$. Since $H_{n-1}$ is a minimal normal subgroup of $G, H_{n-1}=\bigoplus_{i=1}^{m} S_{i}$ where the $S_{i}$ are mutually isomorphic simple groups. Moreover $K \cong G / H$ is also a simple group by maximality of $H$. Let $G_{n-i}=H_{n-i}+K$ for all $i=1,2, \cdots, n$. The centralizer $C_{K}\left(H_{n-1}\right)$ is a normal subgroup of $K$, and so must be 0 or $K$.

Case I: Assume $C_{K}\left(H_{n-1}\right)=K$.

Let $s$ be the order of $H_{n-1}$ and let $t$ be the order of $K$. Since $s, t$ are relatively prime, there exist integers $u, v$ such that $u s+v t=1$. Now for all $h \in H_{n-1}, k \in K$, $u s(h+k)=u s h+u s k=(1-v t) k=k$. Therefore $p_{n-1}=u s 1 \in I\left(G_{n-1}\right)$, where 1 denotes the identity map, is the desired projection from $G_{n-1}$ to $K$.

Case II: Assume $C_{K}\left(H_{n-1}\right)=0$.

If $H_{n-1}$ is a minimal normal subgroup of $G_{n-1}$, it is unique and so $p_{n-1}: G_{n-1} \rightarrow$ $K$ is in $I\left(G_{n-1}\right)$ by Lemma 2.9. If $H_{n-1}$ is not minimal, then without loss of generality, we assume $Q=S_{1} \oplus S_{2} \oplus \cdots \oplus S_{r}$ with $r<m$ a minimal normal subgroup of $G_{n-1}$. By repeating the argument in Case I, we may assume $Q$ is a minimal normal subgroup of $Q+K$ and $C_{K}(Q)=0$. Therefore the projection $q_{1}: Q+K \rightarrow K$ is in $I(Q+K)$.

Applying the above arguments inductively on the group $\bar{G}_{n-1}=G_{n-1} / Q \cong$ $S_{r+1} \oplus S_{r+2} \oplus \cdots \oplus S_{m}+K$, there exists $q_{2} \in I\left(G_{n-1}\right)$ such that for all $h \in H_{n-1}$, $k \in K$, we have $(h+k) q_{2}=c+k$ for some $c \in Q$. A routine argument yields that $p_{n-1}=q_{2} q_{1} \in I\left(G_{n-1}\right)$ is the desired projection from $G_{n-1}$ to $K$.

Now, let $\bar{G}=G / H_{n-1}$. Then $\bar{H}_{n-2}$ is a minimal normal subgroup of $\bar{G}$. Similar reasoning as in Cases I and II above yields that $\bar{p}_{n-2}: G_{n-2} / H_{n-1} \rightarrow K / H_{n-1}$ is in $I\left(G_{n-2} / H_{n-1}\right)$. Hence there exist maps $p_{n-1}$ and $\nu \in I\left(G_{n-2}\right)$ such that for all $x \in H_{n-1}, h \in H_{n-2}$, and $k \in K$, we have $(x+k) p_{n-1}=k$ and $(h+k) \nu=y+k$ for some $y \in H_{n-1}$. Therefore $(h+k) \nu p_{n-1}=(y+k) p_{n-1}=k$. So $p_{n-2}=\nu p_{n-1} \in$ $I\left(G_{n-2}\right)$ is the desired projection from $G_{n-2}$ to $K$.

Inductively, we conclude that the projection $p=p_{0}: G_{0}=G \rightarrow K$ is in $I(G)$. This completes the proof. 
Note that when coprimeness is not assumed in Proposition 2.10, there is no obvious evidence to ensure the lifting of the I-E property from a maximal normal subgroup $H$ to $G$. In Example 2.2, $A_{5}$ is maximal in $G$, but $G$ is not an I-E group. On the other hand, the I-E condition cannot be inherited by a maximal normal subgroup, in general, as shown in Example 2.8.

In the final result of this section, we do not assume the relatively prime condition on the order of $H$ and $K$.

Theorem 2.11. Let $G$ be a finite group and let $G$ be a semidirect sum of a fully invariant subgroup $H$ and a subgroup $K$. Suppose $H$ is a unique minimal normal subgroup of $G$. If $H$ and $K$ are both $I-E$ groups, then $G$ is an $I-E$ group.

Proof. Note that the assumption on $H$ implies the projection map $p \in I(G)$ by Lemma 2.9.

We first consider the case when $H$ is nonabelian. Since $H$ is a minimal normal subgroup of $G, H$ is characteristically simple and so the automorphism near-ring $A(H)=M_{0}(H)$ by [18, Theorem 10.11]. Moreover, the hypothesis that $H$ is an I-E group together with $A(H)=M_{0}(H)$ implies $I(H)=M_{0}(H)$ and thus $H$ is a finite simple nonabelian group by a result of Frölich [8].

Since $H$ is normal in $G$, the centralizer $C_{G}(H)$ is a normal subgroup of $G$. By uniqueness of $H, C_{G}(H)$ must be contained in $H$ and therefore $C_{G}(H)=0$ because $H$ is simple nonabelian.

Let $\alpha \in \operatorname{End}(G)$ be arbitrary. If $G p \alpha(1-p)=K \alpha(1-p)=0$, then $p \alpha(1-p)=$ $0 \in I(G)$. If $K \alpha(1-p) \neq 0$, let $W=\{k \in K \mid k \alpha(1-p) \neq 0\}$. Observe that $1-\rho_{a} \in I(G, H)$ for all $a \in H$ where $\rho_{a}$ is the inner automorphism of $G$ induced by element $a$. Let $k \neq 0 \in K$. If $k\left(1-\rho_{a}\right)=0$ for all $a \in H$, then $k \in C_{G}(H)=0$ and thus there must exist some $b \in H$ with respect to $k$ such that $k\left(1-\rho_{b}\right) \neq 0$. Hence the hypothesis for Theorem 10.24 in [18] holds for the set $K \backslash\{0\}$. By applying [18] Theorem 10.24], there exists $\eta \in I(G)$ such that

$$
k \eta= \begin{cases}k \alpha(1-p), & \text { if } k \in W \\ 0, & \text { if } k \in K \backslash W\end{cases}
$$

It is now easy to verify that $p \alpha(1-p)=p \eta \in I(G)$ for all $\alpha \in \operatorname{End}(G)$. By Theorem 2.1, $G$ is an I-E group.

Now consider the case when $H$ is abelian. The assumption that $H$ is $\mathrm{I}-\mathrm{E}$ and finite abelian implies that $H$ is a cyclic group of prime order. Moreover, that $H$ is a unique minimal normal subgroup of $G$ implies on the one hand the centralizer $C_{G}(H)=H$ because $C_{G}(H)$ is a normal subgroup of $G$, and on the other hand, any two nonzero normal subgroups $H_{1}, H_{2}$ of $G$ satisfying the commutator $\left[H_{1}, H_{2}\right]=0$ must have $H_{1}=H_{2}=H$.

It is now not difficult to verify that the hypothesis for Theorem 3.5 in [4] holds for the set $K \backslash\{0\}$. By using this theorem, there exists an $\eta \in I(G)$ such that, for any $\alpha \in \operatorname{End}(G)$, we have $k \alpha(1-p)=k \eta$ for all $k \in K$. Hence $p \alpha(1-p)=p \eta \in I(G)$ and thus $G$ is an I-E group by Theorem 2.1 .

As a quick application of the above result, we immediately have the symmetric group $S_{n}$ with $n \geq 5$ and the dihedral group $D_{n}$ of order $2 n$ with $n$ odd are all I-E groups. 


\section{Direct sums of I-E Groups}

Proposition 3.1. Let $G$ be direct sum of normal subgroups $H$ and $K$ of $G$. Then:

(1) If $G$ is an $I-E$ group, then $H$ and $K$ are both $I-E$ groups. In particular, all direct components of an $I-E$ group are $I-E$ groups.

(2) Suppose that both $H$ and $K$ are fully invariant subgroups of $G$. If $H$ and $K$ are both $I-E$ groups, then $G$ is an $I-E$ group.

Proof. (1) Since endomorphisms of $H$ and $K$ extend to endomorphisms of $G$ and elements of $I(G)$ restricted to $H$ and $K$ yield elements of $I(H)$ and $I(K)$, it follows that $H$ and $K$ will be I-E groups if $G$ is an I-E group.

(2) Let $\alpha \in \operatorname{End}(G)$. Since $H$ is fully invariant, $\alpha$ restricted to $H$ gives an endomorphism of $H$. Assuming that $H$ is an I-E group, we may represent $\left.\alpha\right|_{H}$ as a finite sum of inner automorphisms of $H$. Therefore $\left.\alpha\right|_{H} \in I(G)$. Similarly, $\left.\alpha\right|_{K} \in I(G)$. Let $\mu \in I(H), \nu \in I(K)$ such that $\left.\alpha\right|_{H}=\left.\mu\right|_{H}$ and $\left.\alpha\right|_{K}=\left.\nu\right|_{K}$. Note that here $\left.\mu\right|_{K}=\left.1\right|_{K}$ and $\left.\nu\right|_{H}=1_{H}$ where 1 is the identity map on $G$. It is then routine to verify that $\alpha=\mu-1+\nu \in I(G)$.

From Proposition 3.1(1), we know that the direct summand of an I-E group is an I-E group. However, this property does not hold for a fully invariant subgroup. Observe that $V_{4}$ (i.e., Klein 4-group) is a fully invariant subgroup of the I-E group $S_{4}$, but $V_{4}$ is not an I-E group. In Proposition 3.1(2), the requirement that both $H$ and $K$ be fully invariant is not superflous as we can see in the following examples. Recall that an abelian group is I-E if and only if it is cyclic. So a direct sum of cyclic groups is not $\mathrm{I}-\mathrm{E}$ if it is not cyclic.

(1) In the infinite case, consider the group $G$ as the direct sum of the integers $\mathbb{Z}$ and the group $\mathbb{Z}_{2}$ of order 2. $G$ is not an I-E group, but both $\mathbb{Z}$ and $\mathbb{Z}_{2}$ are I-E groups. Here $\mathbb{Z}$ is not a fully invariant subgroup of $G$, but $\mathbb{Z}_{2}$ is an I-E group.

(2) In the finite case, consider $V_{4}$ as the direct sum of two copies of $\mathbb{Z}_{2} . V_{4}$ is not an I-E group but $\mathbb{Z}_{2}$ is an I-E group. However $\mathbb{Z}_{2}$ is not a fully invariant subgroup of $V_{4}$.

Making use of Proposition 3.1, we can reprove the following corollary by C. G. Lyons and G. L. Peterson [10] without using Corollary 2.5.

Corollary 3.2 ([10, Corollary 2.2]). If $G$ is the direct sum of $H$ and $K$ where both $H$ and $K$ are $I-E$ groups and if $(|H|,|K|)=1$, then $G$ is an $I-E$ group.

Proof. We first show that $H$ and $K$ will be fully invariant subgroups of $G$. Pick $h \in$ $H$ and $\alpha \in \operatorname{End}(G)$. Then $h \alpha=a+b$ for some $a \in H$ and $b \in K$. Suppose the order of $H$ is $m$ and the order of $K$ is $n$. Then $0=(m h) \alpha=m(h \alpha)=m a+m b=m b$, which then implies that the order of $b$, say $|b|$, is a factor of $m$. But $|b|$ must divide $n$ and so $|b|$ is a common factor of $m$ and $n$. Therefore $|b|=1$ and $h \alpha=a \in H$. Hence $H$ is a fully invariant subgroup of $G$. Similarly, $K$ is a fully invariant subgroup of $G$. The result then follows from Proposition 3.1.

Corollary 3.3. A finite nilpotent group is an I-E group if and only if all its Sylow subgroups are I-E groups.

Proof. Recall that a finite group is nilpotent if and only if it is the direct sum of its Sylow subgroups. By Proposition 3.1 and Corollary 3.2, a finite nilpotent group is an I-E group if and only if all its Sylow subgroups are I-E groups. 
Theorem 3.4. Let $G$ be a finite group with an abelian normal subgroup $H$ such that the order of $H$ is coprime to its index. If $G / H$ and the centralizer $C_{G}(H)$ are $I-E$ groups, then $G$ is an $I-E$ group.

Proof. By the Schur-Zassenhaus theorem, the complement $K$ of $H$ in $G$ exists. Since $H$ is a normal subgroup of $G$, the centralizer $C_{G}(H)$ is normal in $G$. Note that $C_{G}(H)$ is the direct sum of $H$ and $C_{K}(H)$. By Proposition 3.1(1), $H$ is an I-E group. Since the order of $H$ and $K$ are coprime, the projection map $p: G \rightarrow K$ is in $I(G)$ by [25, Theorem 2]. Also $K \cong G / H$ is an I-E group; therefore $G$ is an I-E group by Corollary 2.5 .

As a corollary, we obtain Theorem 3.2 [10] of C. G. Lyons and G. L. Peterson [10, Theorem 3.2].

Corollary 3.5 ([10, Theorem 3.2]). If $G$ is the semidirect sum of a cyclic normal subgroup $H$ and a cyclic subgroup $K$ where $(|H|,|K|)=1$, then $G$ is an $I-E$ group.

Proof. Note that $C_{K}(H)$, as a subgroup of a cyclic group $K$, is cyclic. $C_{K}(H)$ is an I-E group. Because the order of $H$ and $C_{K}(H)$ are relatively prime, $C_{G}(H)=$ $H \oplus C_{K}(H)$ is an I-E group by Corollary 3.2. Hence $G$ is an I-E group by Theorem 3.4 .

\section{ACKNOWLEDGEMENT}

The author is grateful to his advisor Gary F. Birkenmeier for his valuable suggestions and comments. The author also thanks the referee, Professor G. Mason and Professor G. L. Peterson for many suggestions.

\section{REFERENCES}

1. G. Birkenmeier, H. Heatherly and E. Lee, Prime ideals and prime radicals in near-rings, Mh. Math. 117 (1994), 179-197. MR 95e:16045

2. — Completely prime ideals and radicals in near-rings, Proc. Near-rings and Nearfields conference, Fredericton, 1993, (Y. Fong et al.eds), Kluwer Acad. Publ. (1995), 63-73. MR 96k:16082

3. F. Castagna, Sums of automorphisms of a primary group, Pacific J. Math. 27 (1968), 463-473. MR 38:5920

4. Y. Fong and K. Kaarli, Unary polynomials on a class of groups, Acta Sci. Math. (Szeged) 61 (1995), 139-154. MR 97b:16038

5. Y. Fong and J. D. P. Meldrum, The endomorphism near-rings of the symmetric groups of degree at least five, J. Austral. Math. Soc. 30A (1980), 37-49. MR 81j:16043

6. - The endomorphism near-ring of the symmetric group of degree four, Tamkang J. Math. 12 (1981), 193-203. MR 84a:16066

7. H. Freedman, On endomorphisms of primary abelian groups, J. London Math. Soc. 43 (1968), 305-307. MR 37:1471

8. A. Frölich, The near-ring generated by the inner automorphisms of a finite simple group, J. London Math. Soc. 33 (1958), 95-107.

9. L. Fuchs, Recent results and problems on abelian groups, Topics in abelian groups, Scott, Foresman and Company, Glenview, 1963. MR 30:149

10. C. G. Lyons and G. L. Peterson, Semidirect product of I-E groups, Proc. Amer. Math. Soc. 123 (1995), 2353-2356. MR 95j:16054

11. C. G. Lyons and J. J. Malone, Endomorphism near-rings, Proc. Edinburgh Math. Soc. 17 (1970), 71-78. MR 42:4598

12. - Finite dihedral groups and d. g. near-rings I, Compositio Math. 24 (1972), 305-312. MR 46:7321

13. Finite dihedral groups and d. g. near-rings II, Compositio Math. 26 (1973), 249-259. MR 48:8574 
14. C. G. Lyons and G. Mason, Endomorphism near-rings of dicyclic and generalized dihedral groups, Proc. Roy. Irish Acad. 91A (1991), 99-111. MR 93a:16038

15. J. J. Malone, Generalized quaternion groups and distributively generated near-ring, Proc. Edinburgh Math. Soc. 18 (1973), 235-238. [MR 47:5059]

16. Endomorphism near-rings through the ages, Proc. Near-rings and Near-fields conference, Fredericton, 1993, (Y. Fong et al.eds), Kluwer Acad. Publ. (1995), 31-43. MR 96i:16072

17. J. J. Malone and G. Mason, ZS-Metacyclic groups and their endomorphism near-rings, Mh. Math. 118 (1994), 249-265. MR 95j:16055

18. J. D. P. Meldrum, Near-rings and their links with groups, Pitman, London, Research Notes in Math., 134(1985). MR 88a:16068

19. G. L. Peterson, Finite metacyclic $I-E$ and $I-A$ groups, Comm. Algebra 23 (1995), 4563-4585. MR 97h:20029

20. R. S. Pierce, Homomorphisms of primary abelian groups, Topics in abelian groups, Scott, Foresman and Company, Glenview, 1963. MR 31:1299

21. D. J. S. Robinson, A course in the theory of groups, Springer-Verlag, New York, 1996. MR 96f:20001

22. G. Saad, M. J. Thomsen and S. A. Syskin, Endomorphism nearrings on finite groups, a report, Proc. Near-rings and Near-fields conference, Fredericton, 1993, (Y. Fong et al.eds), Kluwer Acad. Publ. (1995), 227-238. MR 96k:16087

23. R. Stringall, Endomorphism rings of abelian groups generated by automorphism groups, Acta Math. Acad. Sci. Hungar. 18 (1967), 401 - 404. MR 35:5509

24. S. A. Syskin, On annihilators in endomorphism nearrings, Comm. Algebra 22 (1994), 57095714. MR 95j:16056

25. Projection endomorphisms on finite groups, Algebra and Logic 34 (1995), 306-310. MR 97d:20021

Department of Mathematics, University of Louisiana at Lafayette, Lafayette, LOUISIANA 70504-1010

E-mail address: fxh2858@usl.edu 